
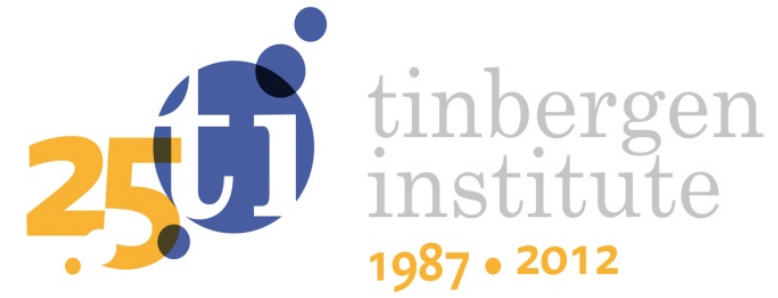

\title{
Players Indifferent to Cooperate and Characterizations of the Shapley Value
}

Conrado Manue/r

Enrique Gonzalez-Aranguenal

René van den Brink²

1 Universidad Complutense de Madrid;

2 Faculty of Economics and Business Administration, VU University Amsterdam, and Tinbergen Institute 
Tinbergen Institute is the graduate school and research institute in economics of Erasmus University Rotterdam, the University of Amsterdam and VU University Amsterdam.

More TI discussion papers can be downloaded at http://www.tinbergen.nl

Tinbergen Institute has two locations:

Tinbergen Institute Amsterdam

Gustav Mahlerplein 117

1082 MS Amsterdam

The Netherlands

Tel.: +31(0)205251600

Tinbergen Institute Rotterdam

Burg. Oudlaan 50

3062 PA Rotterdam

The Netherlands

Tel.: +31(0)10 4088900

Fax: $+31(0) 104089031$

Duisenberg school of finance is a collaboration of the Dutch financial sector and universities, with the ambition to support innovative research and offer top quality academic education in core areas of finance.

DSF research papers can be downloaded at: http://www.dsf.nl/

Duisenberg school of finance

Gustav Mahlerplein 117

1082 MS Amsterdam

The Netherlands

Tel.: +31(0)20 5258579 


\title{
Players indifferent to cooperate and characterizations of the Shapley value
}

\author{
April 7, 2012 \\ C. MANUEL \\ Dpto. de Estadística e I.O. III. Escuela Universitaria de Estadística. Universidad \\ Complutense de Madrid. Av. Puerta de Hierro s/n. 28040 Madrid (Spain). \\ email: conrado@estad.ucm.es \\ E. GONZÁLEZ-ARANGÜENA. \\ Dpto. de Estadística e I.O. III. Escuela Universitaria de Estadística. Universidad \\ Complutense de Madrid. Av. Puerta de Hierro s/n. 28040 Madrid (Spain). \\ email: egaran@estad.ucm.es \\ R. VAN DEN BRINK \\ Department of Econometrics and Tinbergen Institute, VU University, De Boelelaan \\ 1105,1081 HV Amsterdam, The Netherlands. \\ email: jrbrink@feweb.vu.nl
}

\begin{abstract}
In this paper we provide new axiomatizations of the Shapley value for TU-games using axioms that are based on relational aspects in the interactions among players. Some of these relational aspects, in particular the economic or social interest of each player in cooperating with each other, can be found embedded in the characteristic function. We define a particular relation among the players that it is based on mutual indifference. The first new axiom expresses that the payoffs of two players who are not indifferent to each other are affected in the same way if they become enemies and do not cooperate with each other anymore. The second new axiom expresses that the payoff of a player is not affected if players to whom it is indifferent leave the game. We show that the Shapley value is characterized by these two axioms together with the well-known efficiency axiom. Further, we show that another axiomatization of the Shapley value is obtained if we replace the second axiom and efficiency by the axiom which applies the efficiency condition to every class of indifferent players. Finally, we extend the previous results to the case of weighted Shapley values.
\end{abstract}

Keywords TU-game, Shapley value, axiomatization, indifferent players, weighted Shapley values.

MSC 91A06, 91A12, 91A43. 


\section{Introduction}

A cooperative game with transferable utility (a TU-game) is a pair $(N, v)$, $N \subset \mathbb{N}$ being the finite set of players and $v: 2^{N} \rightarrow \mathbb{R}$ with $v(\emptyset)=0$, the characteristic function of the game, that is a real valued map that assigns to each coalition $S \subseteq N$ the worth $v(S)$ that its members can obtain by cooperating. The worth $v(S)$ represents the economic possibilities of the coalition $S$ if it is formed. Shapley (1953a) introduced a point solution for this type of games which is remarkable for its intuitive definition and also for its characterization in terms of a set of reasonable axioms. The relevance of the Shapley value is even greater if we consider the difficulty to axiomatically construct a unique solution from the very limited information about the players interactive environment that is reflected by the characteristic function. From this starting point, the importance and versatility of the value have always been increasing, in part due to multiple approaches to it, from different angles, to obtain characterizations that permit us to understand the value in a greater depth. Several of these axiomatic characterizations (on the set of all TU-games) appear, for example, in Myerson (1980), Young (1985,1994), Hart and Mas-Colell (1989), Chun (1989), Hamiache (2001), van den Brink (2001) or Kongo et al. (2007). Dubey (1975) characterized it in the important subclass of simple games, Neyman (1989) on the additive class spanned by a single game, Algaba et al. (2003) on the class of TU-games defined on antimatroids, Grabish and Lange (2007) on multichoice games and Khmelnitskaya and Yanovskaya (2007) on games with coalition struture. Albizuri (2010) adapts the axiomatic characterization of the Myerson value to define an extension of the Shapley value to cooperative games with externalities.

Winter (2002) is a remarkable survey on the Shapley value, that focuses on technical aspects, like axiomatization. Moretti and Patrone (2008) presents an excellent collection of applications.

In this paper we propose two new axiomatic characterizations of the Shapley value that try to emphasize the importance in this solution of relational aspects in the interactions among players. Some of these relational aspects, in particular the economic or social interest of each player in cooperating with each other, can be found embedded in the characteristic function, if we act as a dog digging up a bone. Even though a TU-game is by definition cooperative, the tendency of players to form coalitions with other players in a given game can be variable. For instance, players in a game are indifferent to cooperate with a dummy player as this cooperation does not include an economic incentive. But even further, suppose that for any coalition that contains players $i$ and $j$ it holds that this coalition can be partitioned in two subcoalitions such that $i$ and $j$ are in different elements of this partition, and the worth of the full coalition is equal to the sum of the worths of the two subcoalitions. Then in some sense also $i$ and $j$ are indifferent to each other's cooperation. This indifference disappears for a pair of players if, roughly speaking, at least in one union of two disjoint coalitions, each of the two players belonging to a different one, this additivity does not hold. Given a game we can, using this idea, classify each pair of players as indifferent or not to the mutual cooperation. 
We consider two axioms concerning this indifference. The first one establishes an equity principle in the spirit of the fairness axioms of Myerson (1977) and van den Brink (2001):

If two players that are not indifferent become enemies during their interaction in the game, and consequently they decide not to cooperate, the resulting payoff for both must be equally affected.

The second axiom points out another aspect that is frequently assumed in social or economic relations:

If two persons are indifferent to cooperate in a certain game, each of them will not be affected by the fact that the other leaves the game. In other words, the contributions of indifferent players must be null (and thus balanced).

The main result of this paper is that, if efficiency and both previous principles are assumed, we must agree with the idea of sharing the value of the grand coalition, $v(N)$, among the players as proposed by the Shapley value.

We also propose another axiom which states that each maximal group of no indifferent players exactly shares its own worth among its members (without any externality). This axiom is related to component efficiency of solutions for graph-games in Myerson (1977). Replacing efficiency and the second new axiom mentioned above by this last axiom, we obtain another characterization of the Shapley value.

Finally, we adapt these axiomatizations to the case of the weighted Shapley values introduced by Shapley (1953b) and Kalai and Samet (1987).

The remainder of this paper is organized as follows. In Section 2 we introduce the notation and some preliminaries. Section 3 contains the main results, the proposed characterizations of the Shapley value. In Section 4 we generalize these characterizations to the case of weighted Shapley values. The last section includes some final remarks, relating the properties used in these characterizations with corresponding ones that appear in other characterizations.

\section{Preliminaries}

When there is no ambiguity about the set of players, we will identify the game $(N, v)$ with its characteristic function $v$. By $G^{N}$ we denote the set of all TUgames with players set $N$, and let $G=\bigcup_{n=1}^{\infty} G^{N}$. $G^{N}$ is an $2^{n}-1$ dimensional real-vector space, where $n=|N|$ denotes the cardinality of the set $N$. A particularly useful basis of this space, the unanimity basis, is the family of games $\left\{u_{S}\right\}_{\emptyset \neq S \subseteq N}$ defined as follows: for all $S \subseteq N, S \neq \emptyset$, and all $T \subseteq N$,

$$
u_{S}(T)= \begin{cases}1, & \text { if } S \subseteq T \\ 0, & \text { otherwise. }\end{cases}
$$

As a consequence, every game in $G^{N}$ can be expressed as a linear combination of these unanimity games: 


$$
v=\sum_{\emptyset \neq S \subseteq N} \Delta_{v}(S) u_{S}
$$

where the unanimity coefficients $\left\{\Delta_{v}(S)\right\}_{\emptyset \neq S \subseteq N}$ are known as the Harsanyi dividends (Harsanyi, 1959). For all $S \subseteq N, \sum_{T \subseteq S} \Delta_{v}(T)=v(S)$ holds. Given a game $(N, v)$, we define the restriction of this game to the set of players $S$ as a new game $\left(S,\left.v\right|_{S}\right)$ of which the characteristic function is given by $\left.v\right|_{S}(T)=v(T)$ for all $T \subseteq S$.

An allocation rule on $G$ is a function $\psi$ that assigns to every $(N, v)$ in $G$ a real $n$-dimensional vector $\psi(N, v), \psi_{i}(N, v)$ representing the payoff outcome for player $i$ in the game. When there is no possibility of confusion, we will write $\psi(v)$ instead of $\psi(N, v)$. An allocation rule is efficient if for every game it exactly allocates the worth of the "grand coalition", i.e.: $\sum_{i \in N} \psi_{i}(N, v)=v(N)$ for all $(N, v)$ in $G$.

The Shapley value, $\varphi(N, v)$, is a very popular allocation rule defined, for all $i \in N$ :

$$
\varphi_{i}(N, v)=\sum_{S \subseteq N \backslash\{i\}} \frac{s !(n-s-1) !}{n !}[v(S \cup i)-v(S)]
$$

or, alternatively,

$$
\varphi_{i}(N, v)=\sum_{S \subseteq N, i \in S} \frac{\Delta_{v}(S)}{s},
$$

$s$ being the cardinality of $S$.

In the literature various allocation rules known as weighted Shapley values have been studied such as the ones introduced by Shapley (1953b) and Kalai and Samet (1987). Weighted Shapley values are useful when one studies game situations in which, besides their 'roles' in the game, players take asymmetric positions in, for example, a network, such as a communication network or a hierarchy. In Shapley (1953b), for exogenously given positive weights $\lambda_{i}>$ $0, i \in \mathbb{N}$, the weighted Shapley value $\varphi^{\lambda}(N, v)$ is given by ${ }^{1}$

$$
\varphi_{i}^{\lambda}(N, v)=\sum_{\substack{S \subseteq N \\ i \in S}}\left(\frac{\lambda_{i}}{\sum_{j \in S} \lambda_{j}}\right) \Delta_{v}(S) \quad \text { for all } i \in N .
$$

If $\lambda_{i}=\lambda_{j}$ for all $i, j \in N$ then $\varphi^{\lambda}(N, v)=\varphi(N, v)$ for all games $(N, v)$, and thus $\varphi^{\lambda}$ is a generalization of the Shapley value $\varphi$.

Another type of weighted Shapley value has been considered in Kalai and Samet (1987). Although they also consider a fixed player set $N$, we adapt their solutions for a variable player set. We assume that, besides weights $\lambda_{i}>$ $0, i \in \mathbb{N}$, the players are also ordered in the sense that there exists a labeling function $l: \mathbb{N} \rightarrow \mathbb{N}$, where $l(i)$ denotes the 'level' of player $i$. For fixed $N \subset \mathbb{N}$ the ordered partition $\Sigma_{l}^{N}=\left(S^{1}, \ldots, S^{t}\right)$ of $N$ is given by $i \in S^{k}, j \in S^{p}$ with

\footnotetext{
${ }^{1}$ We remark that Shapley (1953b) introduced this value for a fixed player set $N$.
} 
$1 \leq k \leq p \leq t$ if and only if $l(i) \leq l(j)$. For fixed $N \subset \mathbb{N}$, the pair $\omega=\left(\lambda^{N}, \Sigma_{l}^{N}\right)$ with $\lambda^{N} \in \mathbb{R}_{++}^{N}$ given by $\lambda_{i}^{N}=\lambda_{i}, i \in N$, is a weight system for player set $N \subset \mathbb{N}$ as considered in Kalai and Samet (1987). For every $S \subseteq N \subset \mathbb{N}$ we denote $k_{l}^{N}(S)=\max \left\{k \in\{1, \ldots, t\} \mid S \cap S^{k} \neq \emptyset\right\}$, and $K_{l}^{N}(S)=S \cap S_{l}^{k_{l}^{N}(S)}$. The KS-weighted Shapley value $\varphi^{(\lambda, l)}(N, v)$ is given by

$$
\varphi_{i}^{(\lambda, l)}(N, v)=\sum_{\substack{S \subseteq N \\ i \in K_{l}^{N}(S)}}\left(\frac{\lambda_{i}}{\sum_{j \in K_{l}^{N}(S)} \lambda_{j}}\right) \Delta_{v}(S) \text { for all } i \in N .
$$

If $l(i)=l(j)$ for all $i, j \in N$ (i.e. $\left.\Sigma_{l}^{N}=(N)\right)$ then $\varphi^{(\lambda, l)}(N, v)=\varphi^{\lambda}(N, v)$ for all games $(N, v)$ on $N$ and $\lambda_{i}>0, i \in N$. Thus, $\varphi^{(\lambda, l)}$ is a generalization of $\varphi^{\lambda}$ (and thus also a generalization of $\varphi$ ).

\section{New axiomatizations of the Shapley value}

In this section we introduce two new characterizations of the Shapley value using properties related to players indifferent to cooperate.

\subsection{The axioms}

We begin with defining what it means when players are indifferent to each other.

Definition 3.1 Given $v \in G^{N}$ and $i, j \in N$, we say that player $i$ is indifferent to cooperate with player $j$ in the game $v$ if there exists $S_{i} \subset N$ with $i \in S_{i}$ and $j \notin S_{i}$ such that for all $S \subseteq N$,

$$
v(S)=v\left(S \cap S_{i}\right)+v\left(S \cap\left(N \backslash S_{i}\right)\right) .
$$

As this property is obviously symmetrical, whenever it holds, we say that $i$ and $j$ are indifferent to mutual cooperation, or indifferent players for short. A direct consequence of the previous definition is that two players maintain their indifference in a given game if some other players leave that game.

Proposition 3.1 Consider $v \in G^{N}$ and two players $i, j \in N$ that are indifferent to cooperate with each other in $v$. Then, $i$ and $j$ are indifferent to cooperate in the game $\left(T,\left.v\right|_{T}\right)$ for all $T \subseteq N$ such that $i, j \in T$.

Proof As $i, j$ are indifferent in $(N, v)$, there exists $S_{i} \subset N$ with $i \in S_{i}$ and $j \notin S_{i}$ such that for all $S \subseteq N$,

$$
v(S)=v\left(S \cap S_{i}\right)+v\left(S \cap\left(N \backslash S_{i}\right)\right) .
$$

Thus, for $R \subseteq T$,

$$
\begin{gathered}
\left.v\right|_{T}(R)=v(R)=v\left(R \cap S_{i}\right)+v\left(R \cap\left(N \backslash S_{i}\right)\right) \\
=v\left(R \cap T \cap S_{i}\right)+v\left(R \cap T \cap\left(N \backslash S_{i}\right)\right) \\
=v\left(R \cap T \cap S_{i}\right)+v\left(R \cap\left(T \backslash\left(S_{i} \cap T\right)\right)\right) \\
=\left.v\right|_{T}\left(R \cap\left(T \cap S_{i}\right)\right)+\left.v\right|_{T}\left(R \cap\left(T \backslash\left(S_{i} \cap T\right)\right)\right) \text {, with } i \in T \cap S_{i} \text { and } j \notin T \cap S_{i} .
\end{gathered}
$$


In the next definition we introduce the transformation of a game $v \in G^{N}$ if, for any reason, two specific players $i$ and $j$ decide not to cooperate with each other.

Definition 3.2 Given $v \in G^{N}$ and $i, j \in N$ we will define the game $v^{i j} \in G^{N}$ as:

$$
v^{i j}=\sum_{\{i, j\} \nsubseteq S \subseteq N} \Delta_{v}(S) u_{S} .
$$

In the given expression for $v^{i j}$, we are assuming that, when two players decide not to cooperate (for instance, because they become enemies), the dividends $\Delta_{v}(S)$ of all subcoalitions of $N$ to which $i$ and $j$ both belong cannot be realized, and thus disappear when calculating the worth of these coalitions. Note that the game is not modified when players $i$ and $j$ are indifferent, so in that case $v^{i j}=v$. This emphasizes the idea that players who are indifferent in that game, have no interest in cooperating. This result is not obvious from Definition 3.2, but it is straightforward from Corollary 3.1 and Proposition 3.4.

An alternative expression for the characteristic function $v^{i j}$ in terms of $v$ is given in the following proposition.

Proposition 3.2 Given $v \in G^{N}$ and $i, j \in N$, for all $S \subseteq N$,

$$
v^{i j}(S)=v(S \backslash\{i\})+v(S \backslash\{j\})-v(S \backslash\{i, j\})
$$

Proof For $S \subseteq N$,

$$
\begin{gathered}
v^{i j}(S)=\sum_{\{i, j\} \nsubseteq T \subseteq N} \Delta_{v}(T) u_{T}(S) \\
=\sum_{T \subseteq N \backslash\{i\}} \Delta_{v}(T) u_{T}(S)+\sum_{T \subseteq N \backslash\{j\}} \Delta_{v}(T) u_{T}(S)-\sum_{T \subseteq N \backslash\{i, j\}} \Delta_{v}(T) u_{T}(S) \\
=\sum_{T \subseteq S \backslash\{i\}} \Delta_{v}(T)+\sum_{T \subseteq S \backslash\{j\}} \Delta_{v}(T)-\sum_{T \subseteq S \backslash\{i, j\}} \Delta_{v}(T) \\
=v(S \backslash\{i\})+v(S \backslash\{j\})-v(S \backslash\{i, j\}) .
\end{gathered}
$$

This last result tells us that, when players $i$ and $j$ decide not to cooperate, the others will interact with each of them separately, adding the obtained outcomes. Obviously, the last term in (1) appears to avoid the duplication of the value obtained by a coalition in which both $i$ and $j$ are excluded.

Next, we introduce a new property for allocation rules for TU-games, stating that, when two players that are not indifferent become enemies, the effect on their respective payoffs is the same.

Definition 3.3 An allocation rule $\psi$ on $G$ satisfies the enemy players property if, for all $(N, v) \in G$ and all pair of players $i, j \in N$ which are not indifferent in $v$, it holds:

$$
\psi_{i}(v)-\psi_{i}\left(v^{i j}\right)=\psi_{j}(v)-\psi_{j}\left(v^{i j}\right) .
$$


Remark 3.1 The restriction to pairs of no indifferent players in the previous definition can be avoided and the property still holds because, if $i$ and $j$ are a pair of indifferent players in $v$ then, $v$ and $v^{i j}$ coincide, as previously established.

Remark 3.2 The enemy players property can be viewed as a weaker version of the fairness property introduced by van den Brink (2001). An allocation rule $\psi$ on $G$ satisfies the fairness property if, for all $(N, v) \in G$, all $i, j \in N$ and all $(N, w) \in G$ in which $i$ and $j$ are symmetric players ${ }^{2}, \psi_{i}(v+w)-\psi_{i}(v)=$ $\psi_{j}(v+w)-\psi_{j}(v)$. The enemy players property is obtained from fairness if we restrict ourselves to pairs of players $i$ and $j$ that are not indifferent in $v$, and the game that is added to $v$, is $w=-\sum_{\{i, j\} \subseteq S \subseteq N} \Delta_{v}(S) u_{S}$. Note that $i$ and $j$ are symmetric in $w$.

The second new property for allocation rules for TU-games states that, if one player $i$ is indifferent to cooperate with another player $j$, then he is even indifferent to the fact that this player leaves the game.

Definition 3.4 An allocation rule $\psi$ defined on $G$ satisfies the null contributions for indifferent players property if, for all $v \in G^{N}$ and all pairs $i, j$ of indifferent players in $v$, it holds:

$$
\psi_{i}(v)-\psi_{i}\left(\left.v\right|_{N \backslash\{j\}}\right)=0 .
$$

Remark 3.3 An allocation rule $\psi$ on $G$ satisfies the balanced contributions property (Myerson, 1980) if, for all $(N, v) \in G$ and all $i, j \in N$ :

$$
\psi_{i}(v)-\psi_{i}\left(\left.v\right|_{N \backslash\{j\}}\right)=\psi_{j}(v)-\psi_{j}\left(\left.v\right|_{N \backslash\{i\}}\right) .
$$

The null contributions for indifferent players property (see Definition 3.4) establishes that, for indifferent players, the contributions must be balanced and, moreover, null. Therefore, in some sense it is more restrictive than balanced contributions as it determines the value of the contributions, but in another sense it is less demanding because it only restricts the contributions for pairs of players that are indifferent.

This property can be extended to the case in which a given player is indifferent to all the players in a group.

Proposition 3.3 Let $(N, v) \in G$ and $T \subseteq N$. Suppose $i \in N \backslash T$ is indifferent to each player in $T$. If $\psi$ is an allocation rule defined on $G$ satisfying the null contributions for indifferent players property, then $\psi_{i}(v)=\psi_{i}\left(\left.v\right|_{N \backslash T}\right)$.

Proof Suppose $T=\left\{j_{1}, j_{2}, \ldots, j_{t}\right\}$. As $\psi$ satisfies the null contributions for indifferent players property, then, iteratively using Proposition 3.1, we have:

$$
\psi_{i}(v)=\psi_{i}\left(\left.v\right|_{N \backslash\left\{j_{1}\right\}}\right)=\psi_{i}\left(\left.v\right|_{N \backslash\left\{j_{1}, j_{2}\right\}}\right)=\ldots=\psi_{i}\left(\left.v\right|_{N \backslash\left\{j_{1}, j_{2}, \ldots, j_{t}\right\}}\right) .
$$

\footnotetext{
${ }^{2}$ Two players $i$ and $j$ are symmetric in game $v$ if $v(S \cup\{i\})=v(S \cup\{j\})$ for all $S \subseteq N \backslash\{i, j\}$.
} 


\subsection{An equivalence relation}

In order to prove the main result in this section, we introduce an equivalence relation induced by each TU-game on its set of players. Moreover, we will prove that this relation coincides with the non-indifference of players.

Definition 3.5 Given $(N, v) \in G^{N}$, we will say that $i, j \in N$ are related in $(N, v)$ if and only if $i=j$ or there exists a family of subsets of $N,\left\{T_{l}\right\}_{l=1}^{m}$, verifying

i) $\Delta_{v}\left(T_{l}\right) \neq 0$ for all $l=1,2, \ldots, m$.

ii) $i \in T_{1}, j \in T_{m}$ and $T_{l} \cap T_{l+1} \neq \emptyset$ for $l=1,2, \ldots, m-1$.

We will denote $i R_{v} j$ if $i, j \in N$ are related in $(N, v)$. It is easy to see that for each TU-game $(N, v), R_{v}$ is an equivalence relation on the players set $N$. The obvious proof is omitted. Let $N / v=\left\{C_{1}, C_{2}, \ldots, C_{k}\right\}$ be the quotient set of $N$ induced by the equivalence relation $R_{v}$. That is, two players $i$ and $j$ belong to the same element $C_{t} \in N / v$ if and only if $i R_{v} j$. The next lemma states that all the players belonging to a coalition having a nonzero dividend belong also to the same element of the quotient set.

Lemma 3.1 Let $(N, v) \in G$ and let $N / v=\left\{C_{1}, C_{2}, \ldots, C_{k}\right\}$ be the quotient set of $N$ induced by the equivalence relation $R_{v}$. If $T \subseteq N$ is such that $\Delta_{v}(T) \neq 0$, then there exists an $l \in\{1,2, \ldots, k\}$ such that $T \subseteq C_{l}$.

Proof Suppose $T \subseteq N$ is such that $\Delta_{v}(T) \neq 0$ and there exist $l, m \in$ $\{1,2, \ldots, k\}, l \neq m$, with $T \cap C_{l} \neq \emptyset$ and $T \cap C_{m} \neq \emptyset$. Consider $i \in T \cap C_{l}$ and $j \in T \cap C_{m}$. As $i, j \in T$ and $\Delta_{v}(T) \neq 0, i R_{v} j$ holds. But this is a contradiction with the fact that $i$ and $j$ are in different equivalence classes and so the result is proved.

As an immediate consequence of the previous lemma, we have:

Corollary 3.1 Let $(N, v) \in G$ and let $i, j \in N$ be such that they are not related in $(N, v)$. If $T \subseteq N$ is such that $i, j \in T$, then $\Delta_{v}(T)=0$.

The next lemma states that the worth of any coalition can be calculated componentwise.

Lemma 3.2 Let $(N, v) \in G$ and $N / v=\left\{C_{1}, C_{2}, \ldots, C_{k}\right\}$ be the quotient set induced on $N$ by the relation $R_{v}$. Then, for all $S \subseteq N, v(S)=\sum_{l=1}^{k} v\left(S \cap C_{l}\right)$.

Proof By Lemma 3.1, if $T \subseteq N$ is such that $\Delta_{v}(T) \neq 0$, then there exists an $l \in\{1,2, \ldots, k\}$ such that $T \subseteq C_{l}$. As $\left\{C_{1}, C_{2}, \ldots, C_{k}\right\}$ is a partition of $N$, we have for all $S \subseteq N$ :

$$
v(S)=\sum_{T \subseteq S} \Delta_{v}(T)=\sum_{l=1}^{k} \sum_{T \subseteq S \cap C_{l}} \Delta_{v}(T)=\sum_{l=1}^{k} v\left(S \cap C_{l}\right) .
$$


Lemma 3.3 Let $(N, v) \in G$ and $i \in N$. Suppose that $S_{i} \subseteq N$ is such that $i \in S_{i}$ and, for all $S \subseteq N, v(S)=v\left(S \cap S_{i}\right)+v\left(S \cap\left(N \backslash S_{i}\right)\right)$. Then, given $T \subseteq N$ with $T \cap S_{i} \neq \emptyset$ and $T \cap\left(N \backslash S_{i}\right) \neq \emptyset, \Delta_{v}(T)=0$ holds.

Proof Let $T \subseteq N$ be such that $T \cap S_{i} \neq \emptyset$ and $T \cap\left(N \backslash S_{i}\right) \neq \emptyset$. By the hypothesis, for all $S \subseteq T, v(S)=v\left(S \cap S_{i}\right)+v\left(S \cap\left(N \backslash S_{i}\right)\right)$. On the other hand, using the definition of the Harsanyi dividends and denoting $t=|T|$ and $s=|S|$,

$$
\begin{aligned}
\Delta_{v}(T) & =\sum_{S \subseteq T}(-1)^{t-s} v(S)=\sum_{S \subseteq T}(-1)^{t-s}\left(v\left(S \cap S_{i}\right)+v\left(S \cap\left(N \backslash S_{i}\right)\right)\right) \\
& =\sum_{S \subseteq T}(-1)^{t-s} v\left(S \cap S_{i}\right)+\sum_{S \subseteq T}(-1)^{t-s} v\left(S \cap\left(N \backslash S_{i}\right)\right) .
\end{aligned}
$$

But $\sum_{S \subseteq T}(-1)^{t-s} v\left(S \cap S_{i}\right)$ can be rewritten as $\sum_{R \subseteq T \cap S_{i}} \alpha(R) v(R)$ where, if $t_{i}$ is the cardinality of $T \cap\left(N \backslash S_{i}\right)$, for all $R \subseteq T \cap S_{i}$, we have $\alpha(R)=\sum_{l=0}^{t_{i}}(-1)^{t-r-l}\left(\begin{array}{l}t_{i} \\ l\end{array}\right)$.

Thus, $\alpha(R)=(-1)^{t-r}$ if $t_{i}=0$, but it is zero otherwise. Since by hypothesis $t_{i} \geqq 1, \sum_{S \subseteq T}(-1)^{t-s} v\left(S \cap S_{i}\right)=0$ holds.

We can analogously proof that $\sum_{S \subseteq T}(-1)^{t-s} v\left(S \cap\left(N \backslash S_{i}\right)\right)=0$, completing the proof.

The next proposition states that the equivalence relation $R_{v}$ is determined by the indifference between players.

Proposition 3.4 Given $(N, v) \in G$ and $i, j \in N, i R_{v} j$ if and only if $i$ and $j$ are not indifferent players in $v$.

Proof Let us first prove that, if $i$ and $j$ are not indifferent players in $v$, then $i R_{v} j$. Let $N / v=\left\{C_{1}, C_{2}, \ldots, C_{k}\right\}$. Suppose, on the contrary, that $i$ and $j$ are not indifferent players in $v$, and $i$ and $j$ are not $R_{v}$-related. Let $C(i)$ be the equivalence class to which $i$ belongs, and therefore $N \backslash C(i)=\cup_{C_{l} \neq C(i)} C_{l}$. Obviously, $j \in N \backslash C(i)$ since by hypothesis $i$ and $j$ are not $R_{v}$-related. By Lemma 3.2, for all $S \subseteq N$,

$$
\begin{gathered}
v(S)=\sum_{l=1}^{k} v\left(S \cap C_{l}\right)=v(S \cap C(i))+\sum_{C_{l} \neq C(i)} v\left(S \cap C_{l}\right) \\
=v(S \cap C(i))+v\left(S \cap\left(\cup_{C_{l} \neq C(i)} C_{l}\right)\right)
\end{gathered}
$$

and thus $i$ and $j$ are indifferent players in $v$, which gives a contradiction.

Reciprocally, in order to prove that the $R_{v}$-relation implies the non indifference between players, suppose that $i, j \in N$ are such that $i R_{v} j$ but they are indifferent in $(N, v)$. Then, there exists $S_{i} \subseteq N$ with $i \in S_{i}$ and $j \notin S_{i}$, such that for all $S \subseteq N$ :

$$
v(S)=v\left(S \cap S_{i}\right)+v\left(S \cap\left(N \backslash S_{i}\right)\right) .
$$


Since, by hypothesis, $i R_{v} j$, there exists $T_{1}, T_{2}, \ldots, T_{m} \subseteq N$, with $\Delta_{v}\left(T_{l}\right) \neq 0$ for all $l=1,2, \ldots, m$, and such that $T_{l} \cap T_{l+1} \neq \emptyset$ for $l=1,2, \ldots, m-1$. Moreover, $i \in T_{1}$ and $j \in T_{m}$. By Lemma 3.3, if $T \subseteq N$ is such that $\Delta_{v}(T) \neq 0$, then $T \cap S_{i}=\emptyset$ or $T \cap\left(N \backslash S_{i}\right)=\emptyset$ and thus, $T \subseteq S_{i}$ or $T \subseteq N \backslash S_{i}$. As a consequence, since $i \in T_{1} \cap S_{i}, T_{1} \subseteq S_{i}$ and $T_{m} \subseteq N \backslash S_{i}$. Finally, let $l^{*} \in\{1,2, \ldots, m\}$ be the first index such that $T_{l^{*}} \subseteq N \backslash S_{i}$. We have $T_{l^{*}-1} \cap T_{l^{*}}=\emptyset$ which gives a contradiction.

\subsection{The axiomatizations}

Using the previous lemmas and propositions, we obtain the main result of this section.

Theorem 3.1 The Shapley value, $\varphi$, is the unique allocation rule on $G$ satisfying efficiency, the enemy players property and the null contributions for indifferent players property.

Proof It is well-known that the Shapley value satisfies efficiency. The enemy players property follows from Remark 3.2. In order to prove null contributions for indifferent players, suppose that $(N, v) \in G$ and $i, j \in N$ are two indifferent players in that game. Then:

$$
\varphi_{i}(v)=\sum_{S \subseteq N, i \in S} \frac{\Delta_{v}(S)}{s}=\sum_{S \subseteq N \backslash\{j\},} \frac{\Delta_{v}(S)}{s}=\varphi_{i}\left(v_{\mid N \backslash\{j\}}\right),
$$

the second equality holding because of Proposition 3.4 and Corollary 3.1. Thus, $\varphi$ satisfies the null contributions for indifferent players property.

Reciprocally, let us prove the uniqueness. Consider an allocation rule $\psi$ defined on $G$ and satisfying efficiency, the enemy players property and the null contributions for indifferent players property. We must prove that

$$
\psi(N, v)=\varphi(N, v)
$$

for all $(N, v) \in G$. The proof uses induction on $d(N, v)=|\delta(N, v)|$, where

$$
\delta(N, v)=\left\{S \subseteq N \mid \Delta_{v}(S) \neq 0\right\} .
$$

If $d(N, v)=0$, then $v \equiv \boldsymbol{O}$ (i.e. $v(S)=0$ for all $S \subseteq N$ ), and thus every $i \in N$ is indifferent to each player in $N \backslash\{i\}$ (take $S_{i}=\{i\}$ in Definition 3.1). Therefore, by Proposition 3.3, $\psi_{i}(v)=\psi_{i}\left(v_{\mid\{i\}}\right)$ for all $i \in N$. As $v_{\mid\{i\}} \equiv \boldsymbol{O}$, by efficiency, $\psi_{i}(v)=\psi_{i}\left(v_{\mid\{i\}}\right)=0$, and thus $\psi$ coincides with the Shapley value, $\varphi$.

Suppose now, by the induction hypothesis, that $\psi(N, v)=\varphi(N, v)$ for games $(N, v) \in G$ with $d(N, v)=k$, and consider a game $(N, v) \in G$ with $d(N, v)=k+$ 1. Let $i \in N$ and let $C(i)$ be the class in the quotient set $N / v=\left\{C_{1}, C_{2}, \ldots, C_{k}\right\}$ to which $i$ belongs. If $C(i)=\{i\}$, then similar as in the case $\delta(N, v)=0$ above, player $i$ is also indifferent to each player in $N \backslash\{i\}$, and using the same reasoning as before, we have $\psi_{i}(v)=\psi_{i}\left(v_{\mid\{i\}}\right)=v(\{i\})$. But, as the Shapley value also satisfies Proposition 3.3 and efficiency, we have $\varphi_{i}(v)=\varphi_{i}\left(v_{\mid\{i\}}\right)=v(\{i\})$ and thus, for this player both allocation rules yield the same payoff. 
Alternatively, suppose that $i \in N$ is such that the cardinality of $C(i)$ is greater than one, and let $j \in C(i), j \neq i$. By the definition of $R_{v}$, there exists a sequence of players $i_{1}=i, i_{2}, i_{3}, \ldots, i_{r}=j$ with $i_{l} \in C(i)$ for $l=1,2, \ldots, r$ and such that, for each $l=1,2, \ldots, r-1$, there is a $T_{l} \subseteq N$ with $\left\{i_{l}, i_{l+1}\right\} \subseteq T_{l}$ and $T_{l} \in \delta(N, v)$. As $\psi$ satisfies the enemy players property:

$$
\psi_{i_{1}}(v)-\psi_{i_{1}}\left(v^{i_{1} i_{2}}\right)=\psi_{i_{2}}(v)-\psi_{i_{2}}\left(v^{i_{1} i_{2}}\right),
$$

and thus:

$$
\psi_{i_{1}}(v)-\psi_{i_{2}}(v)=\psi_{i_{1}}\left(v^{i_{1} i_{2}}\right)-\psi_{i_{2}}\left(v^{i_{1} i_{2}}\right) .
$$

As $d\left(N, v^{i_{1} i_{2}}\right)<d(N, v)=k+1$, using the induction hypothesis,

$$
\psi_{i_{1}}\left(v^{i_{1} i_{2}}\right)=\varphi_{i_{1}}\left(v^{i_{1} i_{2}}\right) \text { and } \psi_{i_{2}}\left(v^{i_{1} i_{2}}\right)=\varphi_{i_{2}}\left(v^{i_{1} i_{2}}\right),
$$

and therefore:

$$
\psi_{i_{1}}(v)-\psi_{i_{2}}(v)=\psi_{i_{1}}\left(v^{i_{1} i_{2}}\right)-\psi_{i_{2}}\left(v^{i_{1} i_{2}}\right)=\varphi_{i_{1}}(v)-\varphi_{i_{2}}(v),
$$

the last equality holding because the Shapley value satisfies the enemy players property. As a consequence, $\psi_{i_{1}}(v)-\varphi_{i_{1}}(v)=\psi_{i_{2}}(v)-\varphi_{i_{2}}(v)$. Using this previous reasoning iteratively, $\psi_{i}(v)-\varphi_{i}(v)=\psi_{j}(v)-\varphi_{j}(v)$ for $j \in C(i)$. Thus, there exists $h_{C(i)} \in \mathbb{R}$ such that $\psi_{j}(v)-\varphi_{j}(v)=h_{C(i)}$ for all $j \in C(i)$.

Finally, using Proposition 3.3. and the null contributions for indifferent players property:

$$
\psi_{j}(v)=\psi_{j}\left(v_{\mid C(i)}\right) \text { for all } j \in C(i),
$$

and thus:

$$
\begin{aligned}
|C(i)| h_{C(i)}= & \sum_{j \in C(i)}\left[\psi_{j}(v)-\varphi_{j}(v)\right]=\sum_{j \in C(i)} \psi_{j}(v)-\sum_{j \in C(i)} \varphi_{j}(v) \\
& =\sum_{j \in C(i)} \psi_{j}\left(v_{\mid C(i)}\right)-\sum_{j \in C(i)} \varphi_{j}\left(v_{\mid C(i)}\right) .
\end{aligned}
$$

By efficiency of both rules $\psi$ and $\varphi$, this last expression is equal to zero and thus, $h_{C(i)}=0$. Therefore $\psi_{i}(v)=\varphi_{i}(v)$, which completes the proof.

The previous proof inspires another characterization of the Shapley value. Let us introduce a variation in the efficiency property in order to include the eventual indifference to cooperate of certain groups of players in a given game.

Definition 3.6 We say that an allocation rule $\psi$ defined on $G$ satisfies efficiency in classes of no indifferent players if for all $(N, v) \in G$ with $N / v=$ $\left\{C_{1}, C_{2}, \ldots, C_{k}\right\}, \sum_{i \in C_{l}} \psi_{i}(v)=v\left(C_{l}\right)$ for all $l=1,2, \ldots, k$.

Note that this property implies efficiency and the null player property, the last property meaning that null players (i.e., players whose marginal contribution to any coalition is zero) earn a zero payoff. If this kind of efficiency in classes is assumed, each maximal group of no indifferent players will share its worth among its members without any transfer of utility to the remaining players. 
Theorem 3.2 The Shapley value $\varphi$, is the unique allocation rule on $G$ satisfying efficiency in classes of no indifferent players and the enemy players property.

Proof It is already proved that $\varphi$ satisfies the enemy players property. So, let us prove that $\varphi$ satisfies efficiency in classes of no indifferent players (shortly called efficiency for indifferent classes). Effectively, given $(N, v) \in G$ with $N / v=\left\{C_{1}, C_{2}, \ldots, C_{k}\right\}$, for each $l=1,2, \ldots, k$ we have:

$$
\sum_{i \in C_{l}} \varphi_{i}(v)=\sum_{i \in C_{l}} \sum_{S \subseteq N, i \in S} \frac{\Delta_{v}(S)}{s}=\sum_{\substack{i \in C_{l} \\ v\left(C_{l}\right),}} \sum_{S \subseteq C_{l}, i \in S} \frac{\Delta_{v}(S)}{s}=\sum_{S \subseteq C_{l}} \Delta_{v}(S)=
$$

the second equality holding because of Corollary 3.1. The proof of the uniqueness follows similar lines of the corresponding one in Theorem 3.1, but using efficiency in classes in each step of the induction. Therefore, it is omitted.

Note that the axioms used in Theorem 3.2 do not use any variation in the player set. Therefore, instead of on $G$ this axiomatization could have been stated on the class $G^{N}$ of all games with any fixed player set $N$. (Note that the proof needs to be adapted).

\section{Weighted Shapley values}

It is well-known that the weighted Shapley values are efficient. Moreover, by Corollary 3.1 it is straightforward to verify that they satisfy the stronger efficiency in classes of no indifferent players and, with Proposition 3.4, also satisfy null contributions for indifferent players. They also satisfy some kind of enemy players property which takes account of the weights.

Definition 4.1 Let the weights $\lambda_{i}>0, i \in \mathbb{N}$, be given. We say that an allocation rule $\psi$ on $G$ satisfies the $\lambda$-enemy players property if, for all $(N, v) \in$ $G$ and all pairs of players $i, j \in N$ which are not indifferent in $v$, it holds: $\psi_{i}(N, v)-\psi_{i}\left(N, v^{i j}\right)=\frac{\lambda_{i}}{\lambda_{j}}\left(\psi_{j}(N, v)-\psi_{j}\left(N, v^{i j}\right)\right)$.

Note that if $\lambda_{i}=\lambda_{j}$ for all $i, j \in \mathbb{N}$, this boils down to the standard enemy players property of Section 3. In a similar way as Theorems 3.1 and 3.2 , it can be shown that the weighted Shapley value $\varphi^{\lambda}(N, v)$ is characterized as follows. ${ }^{3,4}$

Theorem 4.1 Let the weights $\lambda_{i}>0, i \in \mathbb{N}$, be given.

- (i) The weighted Shapley value, $\varphi^{\lambda}$, is the unique allocation rule on $G$ satisfying efficiency, the $\lambda$-enemy players property and null contributions for indifferent players.

\footnotetext{
${ }^{3}$ Below, statement (ii) of Theorem 4.1 can simply be stated for games with a fixed player set. Statement (i), however, uses null contributions for indifferent players and therefore only holds for a variable player set.

${ }^{4}$ The full proof of Theorem 4.1 can be obtained from the authors on request.
} 
- (ii) The weighted Shapley value, $\varphi^{\lambda}$, is the unique allocation rule on $G$ satisfying efficiency in classes of no indifferent players and the $\lambda$-enemy players property.

Considering now the case of the KS-weighted Shapley value, by Corollary 3.1 and Proposition 3.4, also this KS-weighted Shapley value satisfies efficiency in classes of no indifferent players (and thus efficiency) and null contributions for indifferent players. We need to adapt the enemy player property taking account of both $\lambda$ and $\Sigma_{l}^{N}$ as follows.

Definition 4.2 Let the weights $\lambda_{i}>0, i \in \mathbb{N}$, and labeling function $l: \mathbb{N} \rightarrow \mathbb{N}$ be given. We say that an allocation rule $\psi$ on $G$ satisfies the $(\lambda, l)$-enemy players property if, for all $(N, v) \in G$ and all pairs of players $i \in S^{k}, j \in S^{p}$ which are not indifferent in $v$, it holds:

- $\psi_{i}(N, v)-\psi_{i}\left(N, v^{i j}\right)=\frac{\lambda_{i}}{\lambda_{j}}\left(\psi_{j}(N, v)-\psi_{j}\left(N, v^{i j}\right)\right)$ if $k=p$

- $\psi_{i}(N, v)-\psi_{i}\left(N, v^{i j}\right)=0$ if $k<p$.

In a similar way as before, we can show the following.

Theorem 4.2 Let the weights $\lambda_{i}>0, i \in \mathbb{N}$, and $l: \mathbb{N} \rightarrow \mathbb{N}$ be given.

- (i) The KS-weighted Shapley value, $\varphi^{(\lambda, l)}$, is the unique allocation rule on $G$ satisfying efficiency, the $(\lambda, l)$-enemy players property and null contributions for indifferent players.

- (ii) The KS-weighted Shapley value, $\varphi^{(\lambda, l)}$, is the unique allocation rule on $G$ satisfying efficiency in classes of no indifferent players and the $(\lambda, l)$ enemy players property.

\section{$5 \quad$ Final Remarks}

This last section is devoted to analyze the relations between the proposed characterizations of the Shapley value and several other ones in the literature. First, it is known that the Shapley value is characterized by efficiency, the null player property, symmetry and additivity. An allocation rule satisfying these last two properties verifies fairness (van den Brink, 2001) and then also satisfies the enemy players property. Nevertheless, a rule satisfying the enemy players and null contributions for indifferent players properties need not verify the null player property, nor symmetry, nor additivity. This can be seen by considering the rule $\gamma$ defined on $G$ by

$$
\gamma_{i}(N, v)=1 \text { if } i=1 \text { and } 0 \text { otherwise. }{ }^{5}
$$

\footnotetext{
${ }^{5}$ Note that all players get zero when $1 \notin N$.
} 
Second, comparing the present characterization with the one in Myerson (1980) in terms of efficiency, symmetry and balanced contributions, we see that the rule $\gamma$ previously defined does not satisfy symmetry, and the rule $\gamma^{\prime}$ defined on $G$ by $\gamma^{\prime}(N, v)=(n, n, \ldots, n)$ verifies symmetry and balanced contributions but does not satisfy null contributions. Young $(1985,1994)$ characterized the Shapley value in terms of efficiency, symmetry and strong monotonicity. Again, $\gamma$ satisfies the enemy players and null contributions properties but does not satisfy symmetry , and $\gamma^{\prime}$ verifies symmetry and strong monotonicity but does not verify null contributions. Finally, the rule defined on $G$ by $\gamma_{i}^{\prime \prime}(N, v)=1$ if it exists $j \in N, j \neq i$, such that $i$ and $j$ are symmetric in $(N, v)$ and $\gamma_{i}^{\prime \prime}(N, v)=$ $\varphi_{i}(N, v)$, otherwise, satisfies symmetry but does not satisfy null contributions.

With respect to the problem of characterizing the Shapley value on subclasses of games, we must note that the present characterizations do not hold in the important class of simple games. The notion of classes of indifferent players permits us to additively decompose each given game. This fact can be used to reduce the volume of calculations associated to the computation of the Shapley value. In this spirit, the multilinear extension of Owen (1972) also admits and additive decomposition.

\section{Acknowledges}

This research has been partially supported by the "Plan National de I+D+i" of the Spanish Goverment under the project MTM 2008-06778-C02-02/MTM

\section{Bibliography}

Albizuri, M.J. (2010) Games with externalities: games in coalition configuration function form. Math. Meth. of Oper. Res., 72; 171-186.

Algaba, E., Bilbao, J.M., van den Brink, R. and A. Jimenez-Losada (2003) Axiomatizations of the Shapley value for cooperative games on antimatroids. Math. Meth. of Oper. Res., 57; 49-65.

Brink, van den, R. (2001) An axiomatization of the Shapley value using a fairness property. Int. J. Game Theory, 30; 309-319.

Chun, Y. (1989) A new axiomatization of the Shapley value. Games and Economic Behavior, 1; 119-130.

Dubey, P. (1975) On the uniqueness of the Shapley value. Int. J. Game Theory, 4; 131-139.

Grabish, M. and F. Lange (2007) Games on lattice, multichoice games and the Shapley value. Math. Meth. of Oper. Res., 65; 156-167.

Hamiache, G. (2001) Associated consistency and Shapley value. Int. J. Game Theory, 30; 279-289.

Harsanyi, J. C. (1959) A bargaining model for cooperative n-person games. In: Contributions to the Theory of Games IV Tucker A. W. and R. D. Luce (eds.), Princeton UP, Princeton, 325-355.

Hart, S. and A. Mas-Colell (1989) Potencial, value and consistency. Econometrica, $57 ; 589-614$. 
Kalai, E., and D. Samet (1987) On Weighted Shapley Values. Int. J. Game Theory, 16; 205-222.

Khmelnitskaya, A. and B. Yanovskaya (2007). Owen coalitional value without additivity axiom. Math. Meth. of Oper. Res., 66; 255-261.

Kongo, T., Funaki, Y. and S. Tijs (2007) New axiomatization and an implementation of the Shapley value. CentER, Discussion Paper 2007-90.

Moretti, S. and F. Patrone (2008) Transversality of the Shapley value. Top, $16 ; 1-41$.

Myerson, R. B. (1977) Graphs and cooperation in games. Math. Oper. Res., 2; 225-229.

Myerson R. B. (1980) Conference structures and fair allocation rules. Int. J. Game Theory, 9; 169-182.

Neyman, A. (1989) Uniqueness of the Shapley value. Games and Economic Behavior, 1; 116-118.

Owen, G. (1972) Multilinear extensions of games. Management Science, 18(5); 64-79.

Shapley, L. S. (1953a) A value for n-person games. In: The Shapley Value, A. E. Roth (ed.), Cambridge UP, 41-48.

Shapley, L.S. (1953b) Additive and Non-Additive Set Functions, Dissertation, Princeton University, Princeton.

Winter, E. (2002) The Shapley value. Chapter 53 in the Handbook of Game Theory, vol. III, R. J. Aumann and S. Hart (eds.), North-Holland, 2025-2054.

Young, H. P. (1985) Monotonic solutions of cooperative games. Int. J. Game Theory, 14; 65-72.

Young, H. P. (1994) Cost allocation, Chapter 34 in the Handbook of Game Theory, vol II, R. J. Aumann and S. Hart (eds.), North-Holland, 1193-1235. 